\title{
Franz-Keldysh effect in epitaxial ZnO thin films
}

G. Bridoux, M. Villafuerte, J. M. Ferreyra, J. Guimpel, G. Nieva, C. A. Figueroa, B. Straube, and S. P. Heluani

Citation: Appl. Phys. Lett. 112, 092101 (2018); doi: 10.1063/1.5010942

View online: https://doi.org/10.1063/1.5010942

View Table of Contents: http://aip.scitation.org/toc/apl/112/9

Published by the American Institute of Physics 


\title{
Franz-Keldysh effect in epitaxial $\mathrm{ZnO}$ thin films
}

\author{
G. Bridoux, ${ }^{1, a)}$ M. Villafuerte, ${ }^{1}$ J. M. Ferreyra, ${ }^{1}$ J. Guimpel, ${ }^{2}$ G. Nieva,${ }^{2}$ C. A. Figueroa,${ }^{1}$ \\ B. Straube, ${ }^{1}$ and S. P. Heluani ${ }^{1}$ \\ ${ }^{1}$ Laboratorio de Física del Sólido, INFINOA (CONICET-UNT), Facultad de Ciencias Exactas y Tecnología, \\ Universidad Nacional de Tucumán, 4000 San Miguel de Tucumán, Argentina \\ ${ }^{2}$ Centro Atómico Bariloche-CNEA, Instituto Balseiro-Universidad Nacional de Cuyo and CONICET, \\ 8400 S. C. Bariloche, Argentina
}

(Received 27 October 2017; accepted 15 February 2018; published online 26 February 2018)

\begin{abstract}
Photoconductance spectroscopy has been studied in epitaxial $\mathrm{ZnO}$ thin films with different thicknesses that range between 136 and $21 \mathrm{~nm}$. We report a systematic decrease in photoconductivity and a red shift in band edge photoconductance spectra when the thickness is reduced. For thinner films, it is found that the effective energy gap value diminishes. By time dependent photoconductivity measurements, we found an enhanced contribution of the slow relaxation times for thicker films. These effects are interpreted in terms of a band-bending contribution where the Franz-Keldysh effect and the polarization of $\mathrm{ZnO}$ play a major role in thinner films. Published by AIP Publishing.
\end{abstract}

https://doi.org/10.1063/1.5010942

In the last few years, there has been an improvement in the fabrication techniques of transition metal-oxide films. ${ }^{1}$ This has allowed the emergence of a new field of interface phenomena in these materials. ${ }^{2}$ In particular, a high mobility $2 \mathrm{D}$ electron gas (2DEG) has been discovered at the interface of the $\mathrm{LaAlO}_{3} / \mathrm{SrTiO}_{3}{ }^{3}$ and $\mathrm{Mg}_{x} \mathrm{Zn}_{1-x} \mathrm{O} / \mathrm{ZnO}$ bilayers. ${ }^{4-6}$ The latter $\mathrm{ZnO}$-based heterostructure is one of the few oxides where the quantum Hall effect $(\mathrm{QHE})^{4}$ and the fractional quantum Hall effect $(\mathrm{FQHE})^{6}$ have been detected. On the other hand, $\mathrm{ZnO}$ is an appealing wide-bandgap semiconductor for optoelectronics ${ }^{7}$ with an energy gap of $E_{G}=3.36 \mathrm{eV}$ and a large exciton binding energy $(\simeq 60 \mathrm{meV}){ }^{8}$

Moreover, the optoelectronic properties of $\mathrm{ZnO}$ are highly sensitive to adsorbed species on its surface. ${ }^{9,10}$ These effects have been extensively studied in the last few years and they are related to a band bending close to the surface. $9,11,12$ Another appealing characteristic of $\mathrm{ZnO}$ is its piezo-electricity, ${ }^{13-15}$ which can dramatically affect its photo-excitation properties. ${ }^{16}$ All these ingredients make $\mathrm{ZnO}$ a suitable material to emulate the complex heterostructures studied in the last few decades based on GaAs. ${ }^{17}$

The present work focuses on the photoconductivity of $\mathrm{ZnO}$ films and how this property and its spectrum start to deviate from the bulk behavior when the thickness is reduced. These effects can be explained considering an increased contribution of the band bending and the Franz-Keldysh effect for thinner films. These contributions are accompanied by an increment of the tensile strain in the $c$-axis direction, as X-ray diffraction measurements confirm.

$\mathrm{ZnO}$ films were grown by pulsed laser deposition (PLD) on (0001) sapphire substrates at $550{ }^{\circ} \mathrm{C}$ with an oxygen pressure of 0.05 mTorr. A Nd:YAG laser operated at a wavelength of $266 \mathrm{~nm}$, a repetition rate of $10 \mathrm{~Hz}$, and an energy density of $2.1 \mathrm{~J} / \mathrm{cm}^{2}$ was used during deposition. The deposition rate was $\sim 0.018 \mathrm{~nm} \mathrm{~s}^{-1}$. For the electrical resistance measurements, the four point probe method has been employed with a current source-nanovoltmeter configuration

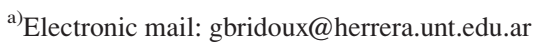

and an applied current of $100 \mu \mathrm{A}$. Indium contacts have been used resulting in linear and symmetric $I-V$ curves. Typical sheet resistances are $R_{S} \simeq 0.4-1 \times 10^{4} \Omega$ with a distance between contacts of $L \simeq 0.5 \mathrm{~mm}$ and a width of $W \simeq 2 \mathrm{~mm}$. Measurements were carried out in a standard cryostat equipped with an optical window, a $1000 \mathrm{~W}$ Xe lamp, and a monochromator (applied incident light from 200 to $800 \mathrm{~nm}$ with an estimated flux density of $\sim 10 \mu \mathrm{W} \mathrm{cm}^{-2}$ ). The photoconductivity spectra have been taken at a scanning speed of $0.1 \mathrm{~nm} \mathrm{~s}^{-1}$ with slower scanning speeds giving the same results.

The X-ray diffraction patterns of the films were obtained using $\mathrm{CuK} \alpha$ radiation $(\lambda=0.15406 \mathrm{~nm})$, and typical results are presented in Fig. 1. The high-angle $\theta-2 \theta$ scan [Fig. 1(a)] and the $\omega$-scan for the (002) peak [Fig. 1(b)] show that the films have grown epitaxially with a wurtzite structure in the 001 direction. The observed Kiessig fringes ${ }^{18}$ in the lowangle $\theta-2 \theta$ reflectivity curve [Fig. 1(c)] are indicative of a film with a uniform thickness and a low roughness.

The room-temperature photoconductivity (PC) spectra of $\mathrm{ZnO}$ films for various thicknesses are shown in Fig. 2(a). In this case, $\mathrm{PC}$ is defined as $P C=\left(\sigma-\sigma_{3.0 \mathrm{eV}}\right) / \sigma_{3.0 \mathrm{eV}}$. The results show that for the thicker film, the onset of PC takes place close to the bulk energy gap value $E_{G} \simeq 3.3 \mathrm{eV}$. As thickness is diminished, the onset $\left(E_{O n}\right)$ shifts to lower energy values, see Fig. 2(a) and inset of Fig. 2(a). For all the spectra, the PC growth is reduced around $\sim 3.5 \mathrm{eV}$ and it is recovered around $\sim 3.8 \mathrm{eV}$. This effect is even more pronounced for the thinner films where in this range of energies, PC decreases.

In addition, PC decreases as the thickness is reduced. In order to confirm this observation, the time dependent photoconductivity (defined in this case relative to $\sigma_{\text {dark }}$ as $P C=(\sigma$ $\left.-\sigma_{\text {dark }}\right) / \sigma_{\text {dark }}$ has been studied for these films. An incident light energy of $\sim 3.3 \mathrm{eV}$ has been applied. The results are summarized in Fig. 2(b). As it has already been noticed, PC decreases as the thickness is diminished. The best fits of these curves were achieved by using a sum of exponential functions, ${ }^{19-22}$ each term of the form: $A_{i} e^{-t / \tau_{i}}$, where $\tau_{i}$ are 

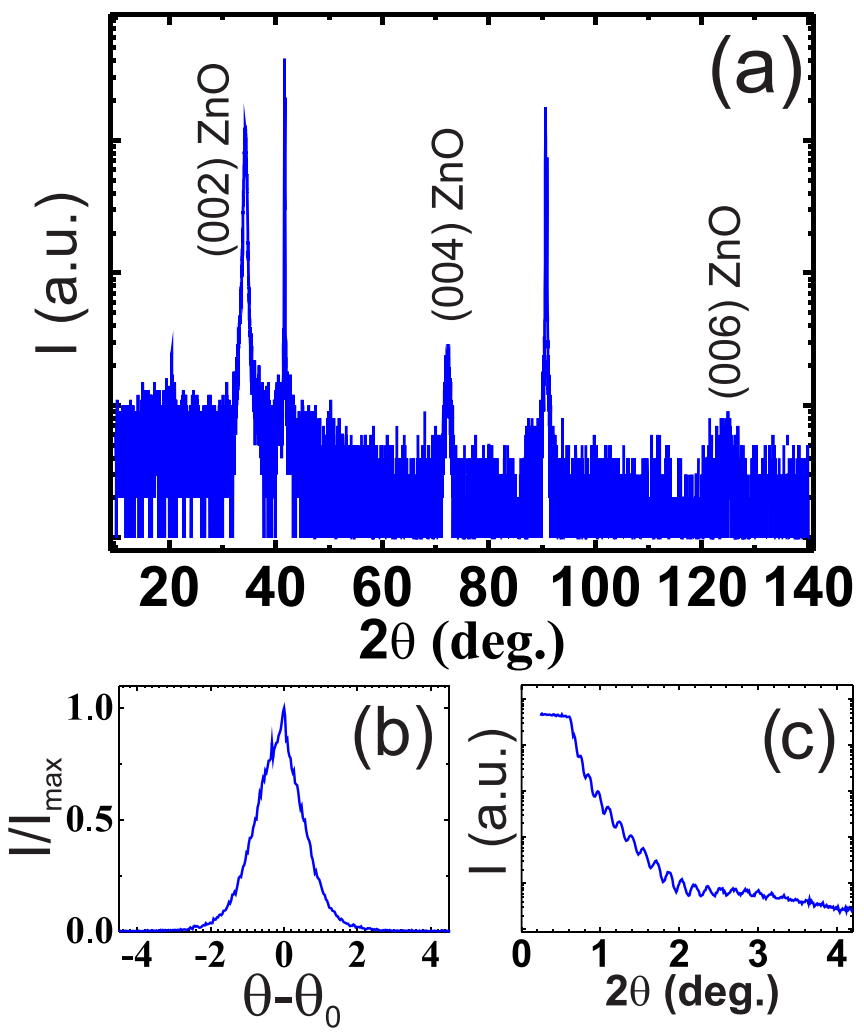

FIG. 1. Typical X-ray diffraction patterns of the fabricated $\mathrm{ZnO}$ films. (a) $\theta-2 \theta$ scan. The characteristics $\mathrm{ZnO}$ peaks are labeled, and the peaks marked with an asterisk correspond to reflections of the sapphire substrate. (b) $\omega$ scan for the (002) peak. A width at half-height of $\sim 1^{\circ}$ is a sign of a highly oriented thin film. (c) Low-angle $\theta-2 \theta$ scan. From this curve, a thickness of $\sim 54 \mathrm{~nm}$ was obtained.

the characteristic relaxation times and $A_{i}$ the corresponding amplitudes with $i=1,2$, and 3 . The results of the fits are presented in Fig. 2(b) and Table I. For the thicker film, the data are well described by the sum of two exponential functions with fast and slow relaxation times. For samples of lower thicknesses, it has been necessary to consider an additional exponential function to fit the data, see Table I. Moreover, as the thickness is reduced, the slowest relaxation time loses weight at the expense of the processes with faster relaxation times, see Fig. 2(b) and Table I. The fact that the photoconducting properties of the films are thickness dependent indicates that surface effects are playing a major role in these phenomena.

The results of Fig. 2 suggest that band bending is a key ingredient to understand these effects. In $\mathrm{ZnO}$, it is well established that oxygen can be adsorbed on its surface ${ }^{23,24}$ producing an accumulation of negative charge on it. This charge accumulation generates an upward band bending of height $\phi$ and width $d,{ }^{9,10}$ see sketch of Fig. 3. These two parameters are related through the expression: ${ }^{9} \phi=e N e d^{2} / 2 \varepsilon_{r} \varepsilon_{0}$, where $e N$ is the effective charge density, $\varepsilon_{r}$ is the relative dielectric constant of $\mathrm{ZnO},{ }^{13}$ and $\varepsilon_{0}$ is the vacuum permittivity. As an estimation, for a charge density of $N \simeq 10^{17} \mathrm{~cm}^{-3}$ and a typical depletion layer of $d \sim 75 \mathrm{~nm}$ for $\mathrm{ZnO},{ }^{10,25,26}$ the resulting $\phi$ is $\sim 0.6 \mathrm{eV}^{26,27}$

When illumination is applied, photo-electrons and holes are generated. These holes tend to move to the surface, where some of them will neutralize the adsorbed ionized oxygen, ${ }^{10,23,24}$ see left panel of Fig. 3. On the other hand,
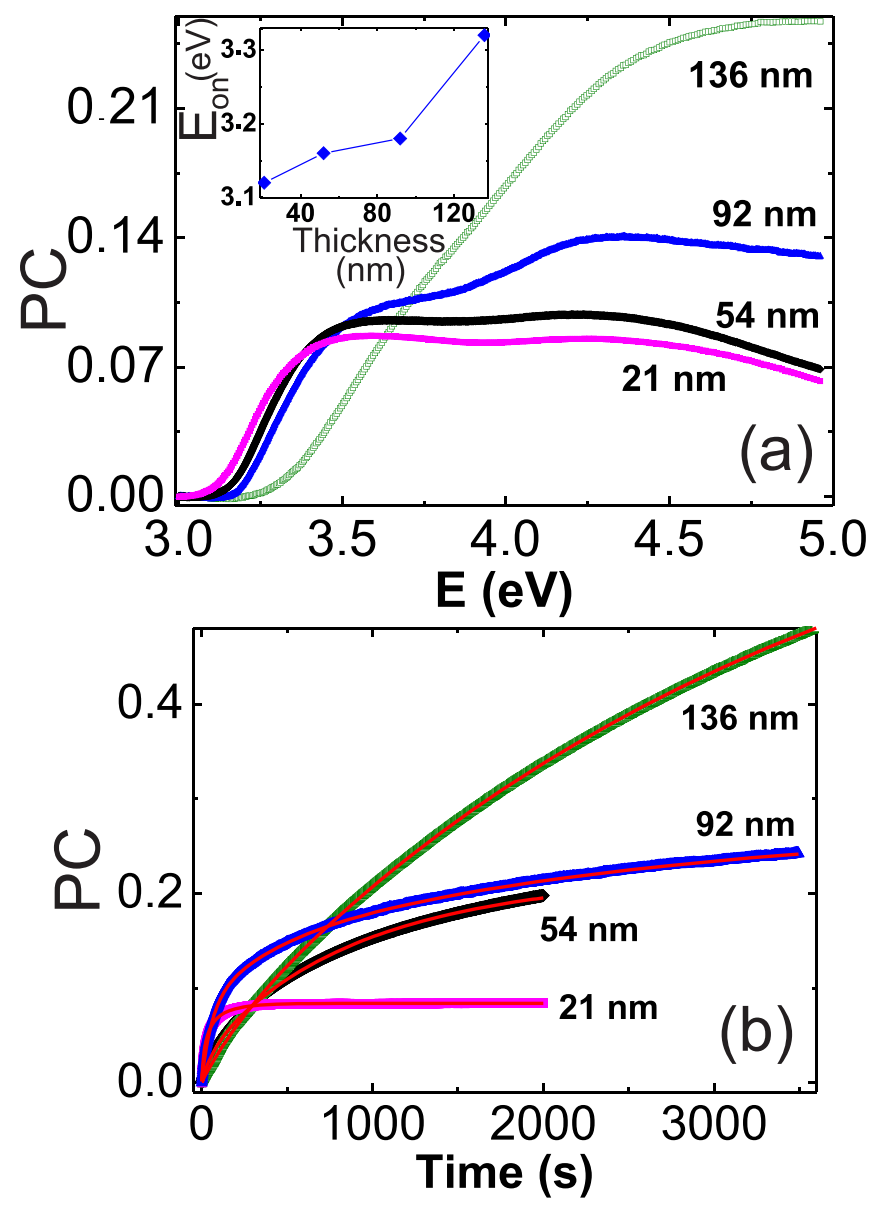

FIG. 2. (a) Photoconductivity of several $\mathrm{ZnO}$ films as a function of the incident light energy for different thicknesses of $136 \mathrm{~nm}$ (green curve), $92 \mathrm{~nm}$ (blue curve), $54 \mathrm{~nm}$ (black curve), and $21 \mathrm{~nm}$ (magenta curve). The inset shows the thickness dependence of the energy onset of these spectra, $E_{O n}$. (b) Time dependent photoconductivity when an incident light energy of $\sim 3.3 \mathrm{eV}$ is applied on the films with thicknesses of $136 \mathrm{~nm}$ (green curve), $92 \mathrm{~nm}$ (blue curve), $54 \mathrm{~nm}$ (black curve), and $21 \mathrm{~nm}$ (magenta curve). The lines are the corresponding exponential fittings (see text). All the measurements have been performed at room-temperature and in air.

electrons are repelled by the upward band bending and they tend to move away from the surface. In the case of the thicker film of $t \simeq 136 \mathrm{~nm}$, where $t>d$ (left panel of Fig. 3), the electrons can move towards the remaining bulk of thickness $t-d$, decreasing the recombination probability and increasing in this way the persistence and $\mathrm{PC} .^{28-30}$ If the film thickness is decreased up to $t \leqq d$ (see central panel of Fig. 3 ), the remaining portion of bulk $t-d$ will be reduced and it will eventually disappear. Without an available bulk region for the photo-electrons, the electron-hole recombination will increase and PC will decrease when the thickness is reduced.

TABLE I. Fitting parameters of the time dependent PC of $\mathrm{ZnO}$ films of various thicknesses by using the sum of exponential decaying functions.

\begin{tabular}{lcccccl}
\hline \hline Thickness $(\mathrm{nm})$ & $\tau_{1}(\mathrm{~s})$ & $\tau_{2}(\mathrm{~s})$ & $\tau_{3}(\mathrm{~s})$ & $\mathrm{A}_{1}$ & $\mathrm{~A}_{2}$ & $\mathrm{~A}_{3}$ \\
\hline 21 & 7 & 30 & 120 & 0.02 & 0.034 & 0.028 \\
54 & 65 & 391 & 1066 & 0.022 & 0.032 & 0.16 \\
92 & 60 & 370 & 2600 & 0.075 & 0.06 & 0.14 \\
136 & 230 & $\mathrm{a}$ & 3440 & 0.032 & $\mathrm{a}$ & 0.68 \\
\hline \hline
\end{tabular}

${ }^{\mathrm{a}}$ The data are well fitted with the sum of two exponential functions. 

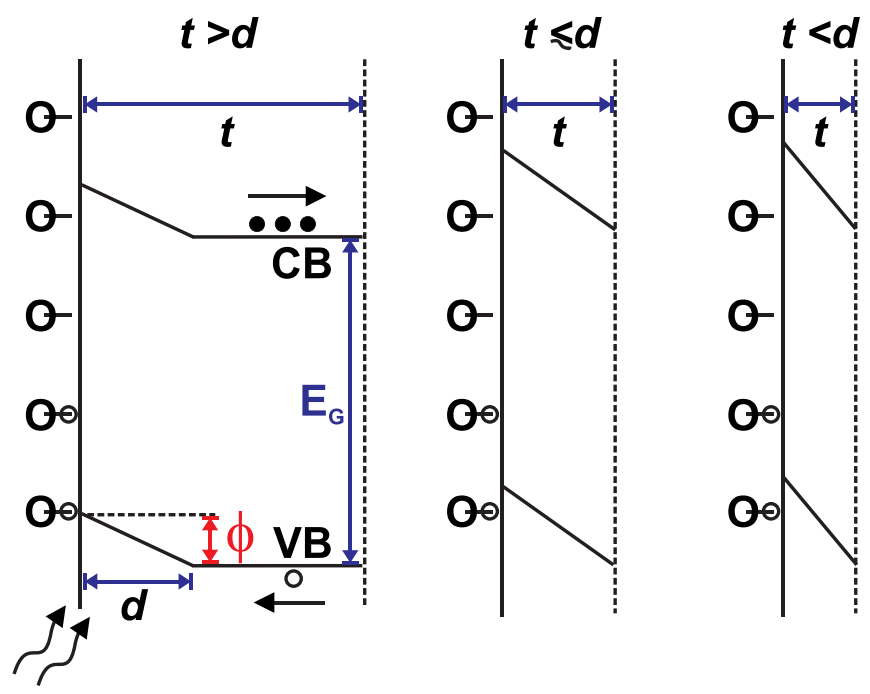

FIG. 3. Energy diagram sketch of the $\mathrm{ZnO}$ films. Adsorbed oxygen on the film surface produces an accumulation of negative charge on it which generates an upward band bending of height $\phi$ and width $d$. When illumination is applied, an electron is promoted from the valence band to the conduction band generating a corresponding hole at the former. Some of the oxygens will be neutralized by these holes.

This is one of the main results of Fig. 2. Since persistence will also decrease compared with the scenario where $t>d$, it is reasonable that the longer relaxation times lose weight as the thickness is lowered, see Fig. 2(b) and Table I.

The reduction of $E_{O n}$ when the thickness is lowered [see Fig. 2(a)] can be understood considering that the FranzKeldysh effect plays a preponderant role in the band bending region of our films. ${ }^{31,32}$ The bending of the valence band (VB) and conduction band (CB) allows that the Airy wavefunction tails of holes and electrons penetrate in the forbidden zone inside the gap. In this way, the effective gap value is reduced in the band bending region of width $d .^{31-33}$ If $E_{O n}$ is associated with the $E_{G}$ value, it is understandable that for the thicker film, $E_{O n}$ coincides with the $E_{G}$ bulk value [see inset of Fig. 2(a)], since the bulk region of width $t-d$ (free of band bending) is a major contributor (see left panel of Fig. 3).

On the other hand, for the thinner films, the bulk region becomes negligible and the band bending contribution is dominant (see central and right panel of Fig. 3). Consequently, the Franz-Keldysh effect would be more pronounced shifting the gap value to lower energies [see inset of Fig. 2(a)]. As a first approximation, PC is proportional to the optical absorption coefficient $\alpha$. Hence, it is possible to fit the onset region of the PC spectra using a model proposed by Dow and Redfield ${ }^{34}$ for the absorption edge. This model considers the presence of a uniform electric field, a situation which is adequate for the $\mathrm{PC}$ spectra of the thinner films since the band bending is a consequence of an internal electrical field. Moreover, this model takes into account the excitonic transitions which are a dominant contribution in $\mathrm{ZnO}$ even at room temperature. ${ }^{13,33}$ In this model, $P C \propto \alpha \sim e^{-E / \Delta E}$, where $\Delta E$ is related to the electric field $\xi$ via $\Delta E=2(e \hbar \xi)^{2 / 3} / 3 m^{* 1 / 3}, \hbar$ is the reduced Planck constant, and $m^{*}$ is the effective mass of the electron. The results of the fitting in the near onset region are presented in Fig. 4(a). The obtained values for $\Delta E$ increase when the thickness is reduced, see Fig. 4(b). This is consistent with an increment of the electric field $\xi$ [see Fig. 4(c)] and the band bending when the thickness is lowered (see central and right panel of Fig. 3). For comparison purposes, the electric field corresponding to the film of $21 \mathrm{~nm}$ is $\sim 1.6$ times larger than the one of $54 \mathrm{~nm}$.

In order to gather information about this latter effect, we have performed a detailed X-ray scan around the (002) peak of these films, and the results are summarized in Fig. 5. As it can be noticed, the (002) peak position is shifted to lower angles compared with the bulk values $\left(\sim 34.42^{\circ}\right),{ }^{35}$ which indicates a tensile strain in the $c$-axis direction. On the other hand, this peak broadens when the thickness is decreased. From the full width at half maximum (FWHM) of these peaks, $w$ (see Fig. 5), and using the Scherrer equation: $\langle D\rangle=K \lambda /(w \cos \theta)$ with $K \simeq 0.94$ and $\lambda=0.15406 \mathrm{~nm},{ }^{36}$ it is possible to estimate the crystallite size of these films. We obtain $\langle D\rangle \sim 17 \mathrm{~nm}$ and $\langle D\rangle \sim 12 \mathrm{~nm}$ for the $54 \mathrm{~nm}$ and $21 \mathrm{~nm}$-thick films, respectively. This shows that there is no significant change in the grain size with the thickness. In $\mathrm{ZnO}$, point defects like oxygen vacancies (which are present in a higher concentration at grain boundaries, surfaces, or interfaces) generate optical transitions that are typically below $\sim 3.0 \mathrm{eV},{ }^{13,37}$ outside the range of energies studied in this work.

The enlargement of the $c$ lattice parameter observed in the $\mathrm{X}$-ray results can be evaluated calculating the induced strain $\Delta c$ / $c$ via the expression $1 / d_{h k l}^{2}=l^{2} / c^{2}+4\left(h^{2}+k^{2}+h \cdot k\right) / 3 a^{2}$, where $(h, k, l)$ are the Miller indices. The resulting strain is higher for the $21 \mathrm{~nm}$ film $\left(\Delta c / c \simeq 5 \times 10^{-3}\right)$ compared to the $54 \mathrm{~nm}$ one $\left(\Delta c / c \simeq 3 \times 10^{-3}\right)$. Due to the piezo-electric characteristics of $\mathrm{ZnO}$, this tensile strain can induce an electric polarization along the $c$-axis: $P \simeq e_{33} \Delta c / c,{ }^{13-15}$ where $e_{33}$ is the piezo-electric coefficient along the $c$-axis direction. As the thickness is reduced, this induced electric field will increase the

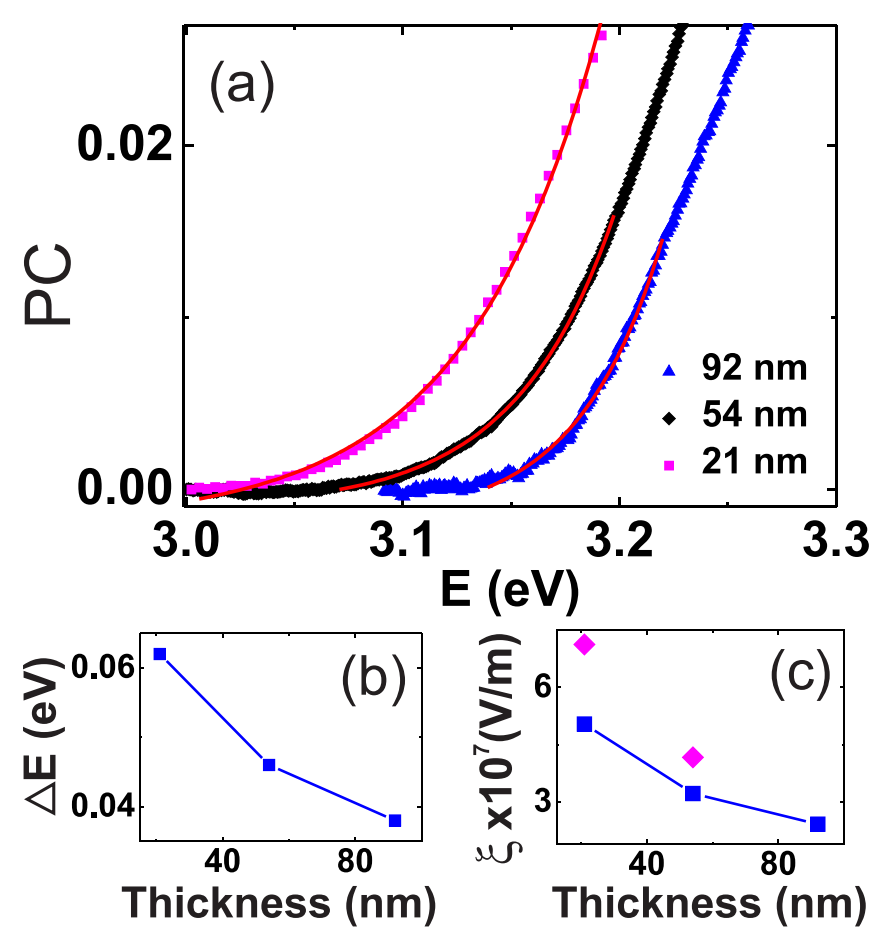

FIG. 4. (a) Fits (red lines) of the photoconductivity spectra using the model of Dow and Redfield. (b) Resulting thickness dependence of the parameter $\Delta E$. (c) Thickness dependence of the electric field obtained from the $\Delta E$ values (blue squares) and the one resulting from the induced electric polarization $\xi_{p}$ (magenta diamonds). 


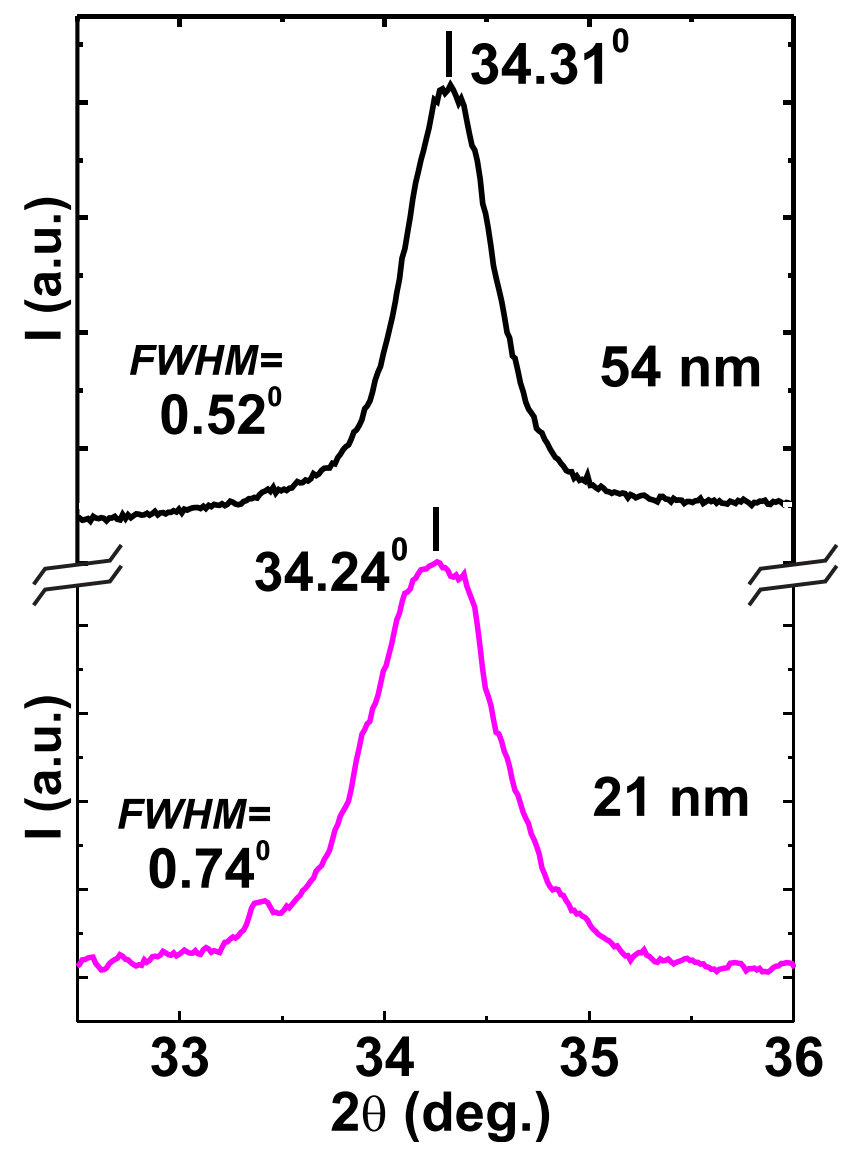

FIG. 5. Detailed $\theta-2 \theta$ X-ray scan around the (002) peak of the ZnO films. Results for the $54 \mathrm{~nm}$ film (black curve) and the $21 \mathrm{~nm}$ one (magenta curve) are presented.

upward band bending due to the increment of $\Delta c / c$ (see central and right panel of Fig. 3). In fact, the ratio $P_{21 \mathrm{~nm}} / P_{54 \mathrm{~nm}}$ gives $\sim 1.6$ in agreement with the corresponding ratio of electric fields obtained from the PC spectra fittings, see Fig. 4(c). Using a typical value of $e_{33} \simeq 1.14 \mathrm{C} \mathrm{m}^{-2}$ for $\mathrm{ZnO}$ (reported values can vary from 0.8 to $\left.1.4 \mathrm{C} \mathrm{m}^{-2}\right),{ }^{13-15}$ it is possible to calculate the electric field due to the induced polarization, $\xi_{p}$. The results are shown in Fig. 4(c). The values obtained for $\xi_{p}$ are very close to the ones extracted from the PC spectra fittings. The differences between these values can be related to a charge redistribution effect that takes place when the electric field $\xi_{p}$ is established. The latter effect will tend to minimize the electrostatic surface energy and stabilize the polar surfaces. ${ }^{38-40}$ It should also be considered that thermal effects on the spectra could be present. This effect can slightly modify the $\xi$ values obtained from PC spectra fittings [see Fig. 4(c)].

On the other hand, for films with $t<d$, PC and the relaxation times, $\tau$, decrease when the thickness is lowered [see Fig. 2(b) and Table I]. In order to get more insight into this effect, we have evaluated the electron and hole wavefunction $\psi_{e, h}$ on the ground state of the CB and VB, respectively. For this purpose, we have made use of the effective mass approximation and we have considered that the overlapping between $\psi_{e}$ and $\psi_{h}$ is inversely proportional to the relaxation time: ${ }^{29}\left\langle\psi_{e} \mid \psi_{h}\right\rangle^{2} \propto \tau^{-1}$. As previously mentioned, we have considered a potential $\phi(z)$ which varies linearly along the thickness direction due to the band bending effect on the $\mathrm{CB}$ and $\mathrm{VB} .{ }^{37}$ We have also assumed that $\phi(z)$ is delimited by an infinite potential at the film surface and at the interface with the substrate, see inset of Fig. 6. The resulting wave-functions are of the form

$$
\psi_{j}(z)=a_{j} A_{i}\left[-\beta_{j}\left(\alpha_{j}-z\right)\right]+b_{j} B_{i}\left[-\beta_{j}\left(\alpha_{j}-z\right)\right],
$$

where $j=e, h$ (electrons or holes), $\mathrm{A}_{i}$ and $\mathrm{B}_{i}$ are the Airy functions, ${ }^{31,37} \beta_{j}=\left(2 m_{j}^{*} e \xi / \hbar^{2}\right)^{1 / 3}$, and $\alpha_{j}=E_{0 j} / e \xi$, with $E_{0 j}$ being the ground state energy. The coefficients $a_{j}$ and $b_{j}$ can be evaluated using the normalization and boundary conditions. In Fig. 6, we compare the results for a thin film of $t=40 \mathrm{~nm}$ (panel a) and another one of $t=21 \mathrm{~nm}$ (panel b). A qualitative analysis shows that while an $\sim 1.6$ times higher electric field for the thinner film tends to decrease $\left\langle\psi_{e} \mid \psi_{h}\right\rangle^{2}$, the confinement of these wave-functions has an opposite effect increasing the probability of recombination and $\left\langle\psi_{e} \mid \psi_{h}\right\rangle^{2}$. The overall result for the thinner film is an enhancement of $\left\langle\psi_{e} \mid \psi_{h}\right\rangle^{2}$ compared with the thicker one. Hence, the relaxation times $\tau$ and $\mathrm{PC}^{41}$ will tend to decrease as the thickness is lowered.
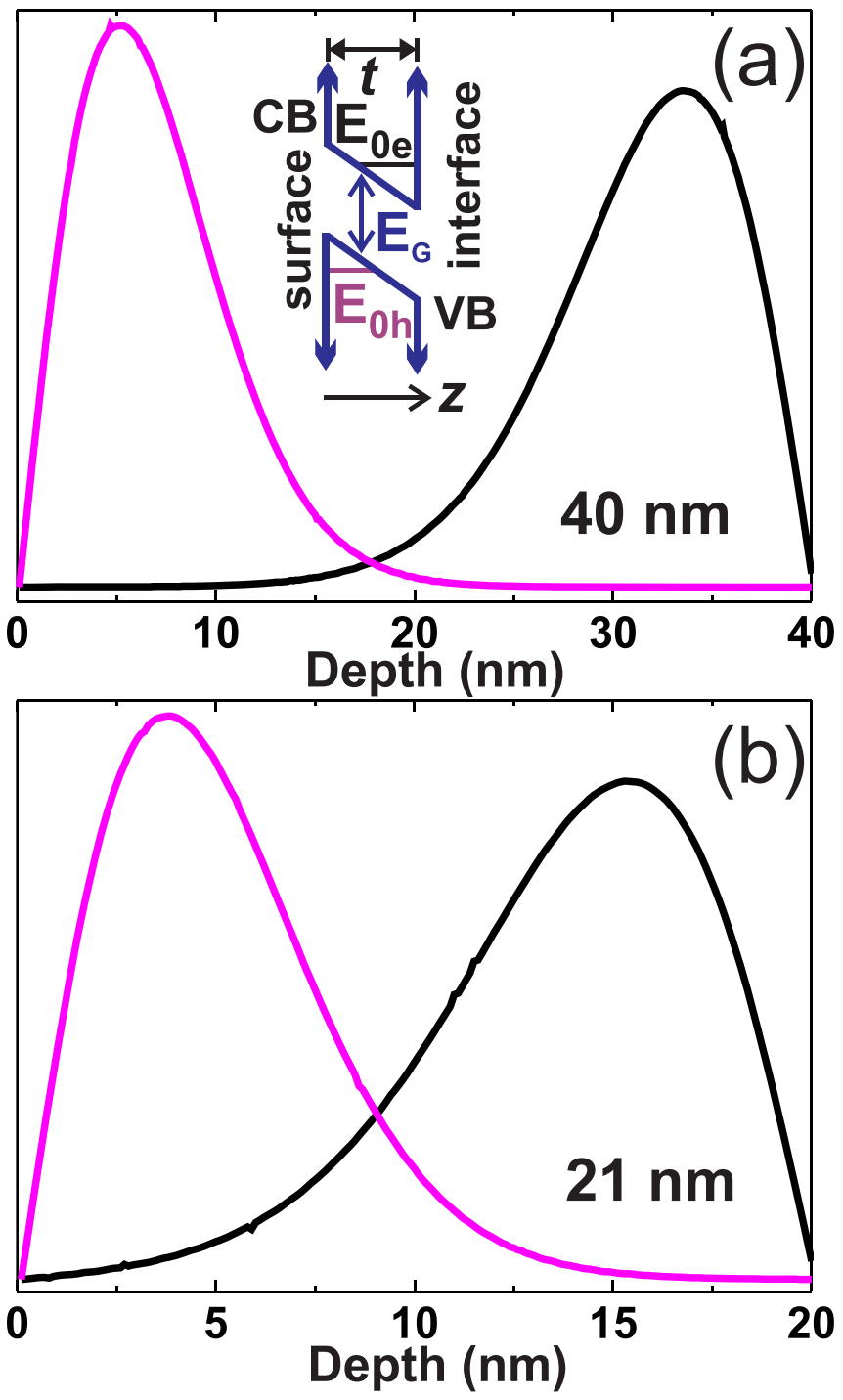

FIG. 6. Electron (black curve) and hole (magenta curve) wave-function amplitudes along the depth direction of the film for: (a) a thickness of $t=40 \mathrm{~nm}$ with an electric field of $\xi=3.2 \times 10^{7} \mathrm{Vm}^{-1}$ and (b) $t=21 \mathrm{~nm}$ with $\xi=5 \times 10^{7} \mathrm{Vm}^{-1}$. The sketch of the inset describes the used potential $\phi(z)$. 
As it has been already pointed out, the energy dependent PC starts to slow down around $\sim 3.5 \mathrm{eV}$, see Fig. 2(a). In other words, there is a mechanism that inhibits photoconduction at this applied incident light energy. In contrast to other semiconductors, there is evidence that very stable phonon-exciton complexes are formed in $\mathrm{ZnO}$ at this energy. ${ }^{42,43}$ In the case of $\mathrm{ZnO}$, a longitudinal optical (LO) phonon with an energy of $\sim 70 \mathrm{meV}^{42-44}$ is involved. The stability of this complex is partially guaranteed by the condition: $a_{x} \leqslant r_{e}+r_{h} \simeq 2.2 \mathrm{~nm}$, where $a_{x} \simeq 1.66 \mathrm{~nm}$ is the exciton radius, and $r_{e}$ and $r_{h}$ are the electron and hole polaron radii, respectively. ${ }^{43}$ When illumination reaches an energy of $\sim 3.5 \mathrm{eV}$, a considerable amount of these dressed excitons are photo-generated. Since these excitons do not contribute to the electrical conduction, they make the photoconductivity to slow down, see Fig. 2(a). At a certain point, if the energy is increased well above this value, the production of these complexes will not be efficient anymore. In this situation, the rate of photo-generated unbound electron-hole pairs will start to dominate again making the photoconductivity to rise again, see Fig. 2(a).

This work was supported by PIP-No. 585, SCAIT-No. 26/E530, PICT-No. 2016-3356, SNMAG and SINALA facilities. GN and JG acknowledge the support from PICTNo. 2014-0164.

\section{${ }^{1}$ J. Heber, Nature 459, 28 (2009).}

${ }^{2}$ H. Y. Hwang, Y. Iwasa, M. Kawasaki, B. Keimer, N. Nagaosa, and Y. Tokura, Nat. Mater. 11, 103 (2012)

${ }^{3}$ A. Ohtomo and H. Y. Hwang, Nature 427, 423 (2004).

${ }^{4}$ A. Tsukazaki, A. Ohtomo, T. Kita, Y. Ohno, H. Ohno, and M. Kawasaki, Science 315, 1388 (2007).

${ }^{5}$ A. Tsukazaki, A. Ohtomo, M. Kawasaki, S. Akasaka, H. Yuji, K. Tamura, K. Nakahara, T. Tanabe, A. Kamisawa, T. Gokmen, J. Shabani, and M. Shayegan, Phys. Rev. B 78, 233308 (2008).

${ }^{6}$ A. Tsukazaki, S. Akasaka, K. Nakahara, Y. Ohno, H. Ohno, D. Maryenko, A. Ohtomo, and M. Kawasaki, Nat. Mater. 9, 889 (2010).

${ }^{7}$ M.-P. Lu, C.-W. Chen, and M.-Y. Lu, Phys. Rev. Appl. 6, 054018 (2016).

${ }^{8}$ D. P. Norton, Y. Heo, M. Ivill, K. Ip, S. Pearton, M. F. Chisholm, and T. Steiner, Mater. Today 7, 34 (2004).

${ }^{9}$ Z. Zhang and J. T. Yates, Jr., Chem. Rev. 112, 5520 (2012).

${ }^{10}$ J. C. Moore and C. V. Thompson, Sensors 13, 9921 (2013).

${ }^{11}$ R. Heinhold, G. T. Williams, S. P. Cooil, D. A. Evans, and M. W. Allen, Phys. Rev. B 88, 235315 (2013).

${ }^{12}$ D. H. Lien, J. R. Durán Retamal, J. J. Ke, C. F. Kang, and J. H. He, Nanoscale 7, 19874 (2015).

${ }^{13} \mathrm{H}$. Morkoç and Ü. Özgür, Zinc Oxide (Wiley-VHC, Weinheim, 2009).
${ }^{14}$ A. Dal Corso, M. Posternak, and R. Resta, and A. Baldereschi. Phys. Rev. B 50, 10715 (1994).

${ }^{15}$ F. Bernardini, V. Fiorentini, and D. Vanderbilt, Phys. Rev. B 56, R10024 (1997).

${ }^{16}$ Y. Liu, Q. Yang, Y. Zhang, Z. Yang, and Z. L. Wang, Adv. Mater. 24, 1410 (2012).

${ }^{17} \mathrm{G}$. Bastard, Wave Mechanics Applied to Semiconductor Heterostructures, Monographies de Physique (Les Éditions de Physique, Les Ulis, 1988).

${ }^{18}$ H. Kiessig, Ann. Phys. 402, 769 (1931).

${ }^{19}$ R. H. Bube, Photoconductivity of Solids (John Wiley \& Sons, New York, 1960).

${ }^{20}$ K. X. Jin, W. Lin, B. C. Luo, and T. Wu, Sci. Rep. 5, 8778 (2015).

${ }^{21}$ R. H. Bube, Photoelectronics Properties of Semiconductors (Cambridge University Press, Cambridge, 1992).

${ }^{22}$ S. A. Studenikin, N. Golego, and M. Cocivera, J. Appl. Phys. 83, 2104 (1998).

${ }^{23}$ E. G. Barbagiovanni, V. Strano, G. Franz, and S. Mirabella, Appl. Phys. Lett. 109, 143104 (2016).

${ }^{24}$ A. J. Gimenez, J. M. Yañez-Limón, and J. M. Seminario, J. Phys. Chem. C 115, 282 (2011).

${ }^{25}$ K. Vanheusden, C. H. Seager, W. L. Warren, D. R. Tallant, and J. A. Voigt, Appl. Phys. Lett. 68, 403 (1996).

${ }^{26}$ L. L. Yang, Q. X. Zhao, M. Q. Israr, J. R. Sadaf, M. Willander, G. Pozina, and J. H. Yang, J. Appl. Phys. 108, 103513 (2010).

${ }^{27}$ Z.-M. Liao, K.-J. Liu, J.-M. Zhang, J. Xu, and D.-P. Yu, Phys. Lett. A 367, 207 (2007).

${ }^{28}$ C. Soci, A. Zhang, B. Xiang, S. Dayeh, D. Aplin, J. Park, X. Bao, Y. Lo, and D. Wang, Nano Lett. 7, 1003 (2007).

${ }^{29}$ J. Singh, Electronic and Optoelectronic Properties of Semiconductors Structures (Cambridge University Press, Cambridge, 2003).

${ }^{30}$ X. Feng, L. Feng, M. Jin, J. Zhai, L. Jiang, and D. Zhu, J. Am. Chem. Soc. 126, 62 (2004).

${ }^{31}$ J. H. Davies, The Physics of Low-Dimensional Semiconductors (Cambridge University Press, New York, 1998).

${ }^{32} \mathrm{C}$. Lamberti, Characterization of Semiconductor Heterostructures and Nanostructures (Elsevier, Amsterdam, 2008).

${ }^{33}$ A. Cavallini, L. Polenta, M. Rossi, T. Stoica, R. Calarco, R. J. Meijers, T. Richter, and H. Lüth, Nano Lett. 7, 2166 (2007).

${ }^{34}$ J. D. Dow and D. Redfield, Phys. Rev. B 1, 3358 (1970).

${ }^{35}$ A. Kuroyanagi, J. Appl. Phys. 66, 5492 (1989).

${ }^{36}$ A. Patterson, Phys. Rev. 56, 978 (1939).

${ }^{37}$ J. M. Ferreyra, G. Bridoux, M. Villafuerte, B. Straube, J. Zamora, C. A. Figueroa, and S. P. Heluani, Solid State Commun. 257, 42 (2017).

${ }^{38}$ C. Noguera, J. Phys.: Condens. Matter 12, R367 (2000).

${ }^{39}$ B. Meyer and D. Marx, Phys. Rev. B 67, 039902 (2003).

${ }^{40}$ B. Meyer, Phys. Rev. B 69, 045416 (2004).

${ }^{41}$ M. Villafuerte, D. J. Zamora, G. Bridoux, J. M. Ferreyra, M. Meyer, and S. P. Heluani, J. Appl. Phys. 121, 064501 (2017).

${ }^{42}$ W. Y. Liang and A. D. Yoffe, Phys. Rev. Lett. 20, 59 (1968).

${ }^{43}$ S. Shokhovets, O. Ambacher, B. K. Meyer, and G. Gobsch, Phys. Rev. B 78, 035207 (2008).

${ }^{44}$ R. Yukawa, K. Ozawa, S. Yamamoto, H. Iwasawa, K. Shimada, E. F. Schwier, K. Yoshimatsu, H. Kumigashira, H. Namatame, M. Taniguchi, and I. Matsuda, Phys. Rev. B 94, 165313 (2016). 